\title{
Matriz de contabilidad social y análisis estructural de una economía rural: el ejido Los Lirios, municipio de Arteaga, Coahuila, México
}

\author{
Gaspar Núñez Rodríguez* y Valentín Mendoza Meza**
}

\section{RESUMEN}

El diseño de políticas de desarrollo regional requiere tanto del conocimiento de la región como de instrumentos analíticos apropiados. Las matrices de contabilidad social regionales cumplen con suficiencia ambos requerimientos, pues además de proporcionar un perfil detallado de la región estudiada, sirven de base a una amplia gama de modelos del análisis estructural, y de modelos multisectoriales en general. Este artículo tiene dos objetivos principales: primero, presentar una matriz de contabilidad social para una economía rural, documentando la metodología utilizada para su construcción, la cual abarca desde la obtención de la base de datos por medio de encuestas directas, hasta la forma de cuadrar la matriz. El segundo objetivo es realizar un análisis estructural básico, utilizando el conocido modelo de multiplicadores generalizados, para analizar los efectos de impactos exógenos seleccionados.

Números de clasificación: JEL: R11, R15, R34.

Palabras clave: matriz de contabilidad social, multiplicadores generalizados, economía rural, desarrollo regional, política económica.

\begin{abstract}
Design of regional development policy, requires knowledge of the region and appropriate analytic tools. Regional Social Accounting Matrices have shown proficiency in both respects, since they supply a detailed profile of the region and, at the same time, constitute the basis of a wide array of models of the structural analysis and multi-sector models in general. This paper has two main goals. First, to present a Social Accounting Matrix of a rural economy, documenting the methodology used to construct it, from collecting data through direct surveys, to matrix squaring. The second goal is to carry out a basic structural analysis, using the well known model of generalized multipliers, to analyze the effects of selected exogenous shocks.
\end{abstract}

Numbers of classification: JEL: R11, R15, R34.

Key words: Social accounting matrix, generalized multipliers, rural economy, regional development.

\footnotetext{
* Investigador del Centro de Investigaciones Socioeconómicas de la Universidad Autónoma de Coahuila.

** Consultor independiente con maestría en economía regional por la Universidad Autónoma de Coahuila.
} 


\section{INTRODUCCIÓN}

El diseño de políticas para el desarrollo regional requiere tanto del conocimiento de la región como de instrumentos analíticos apropiados. Después de más de medio siglo de evolución - entre los primeros trabajos de contabilidad social se cuentan los de Stone (1944) - , las matrices de contabilidad social (MCS) se han consolidado como un instrumento que cumple con suficiencia ambos requerimientos pues, además de proporcionar un perfil detallado de la región bajo estudio, constituyen la base de una amplia gama tanto de modelos del análisis estructural, como de modelos multisectoriales en general.

El presente trabajo tiene dos objetivos principales. El primero es presentar una MCS para una economía rural, documentando la metodología utilizada para su construcción, que abarca desde la obtención de la base de datos mediante encuestas directas, hasta la forma de cuadrar la matriz. El segundo objetivo consiste en realizar un análisis estructural básico de dicha economía utilizando, por un lado, el conocido modelo de multiplicadores generalizados para analizar algunos impactos exógenos, así como el cálculo de los índices de Rasmussen para aportar una clasificación preliminar de los sectores de la economía estudiada.

La elaboración de matrices de contabilidad social en el nivel local, siguiendo la metodología aquí empleada — basada en el trabajo de Yúnez-Naude y Taylor (1999) - , es escasa en nuestro país. Entre los trabajos realizados podemos mencionar el de Adelman, Taylor y Vogel (1987), quienes realizaron un análisis de la economía de una comunidad rural de la región de Pátzcuaro, en el estado de Michoacán. Porotro lado, Yúnez-Naude(1999) llevó a cabo una investigación de las comunidades de Concordia, en el estado de Coahuila; El Chante, en Jalisco; Napízaro, en Michoacán, y San Quintín, en Baja California.

Bracamonte y Méndez (2004) también utilizaron esta metodología para analizar las implicaciones que han tenido las políticas de apoyo en el desarrollo de las comunidades para dos pueblos de la región de Sonora: Trincheras y Arizpe. Respecto al estado de Coahuila se tiene como última referencia el trabajo efectuado por Villa (2001) sobre El Manantial, ejido perteneciente al municipio de Matamoros.

En relación con las aplicaciones del modelo de multiplicadores contables, existe una extensa bibliografía internacional; aquí nos limitaremos a señalar algunos de los trabajos más relevantes para México. ${ }^{1}$ En la esfera nacional están los trabajos de Yúnez-Naude y González (2008), Chapa (2003) y Núñez (2003), En el ámbito regional tenemos los de Domínguez (2008) y Chapa et al. (2008).

\footnotetext{
${ }^{1}$ Agradecemos a un dictaminador anónimo las sugerencias en este punto.
} 
Yúnez-Naude y González (2008) construyen una matriz de contabilidad social para 10 comunidades rurales y aplican la técnica de descomposición de multiplicadores para analizar las actividades productivas de las zonas rurales pobres. Sus principales resultados muestran que la agricultura comercial tiene los efectos multiplicadores más altos en el ingreso y la disminución de la pobreza, y que la producción de cultivos básicos (maíz) tiene grandes enlaces de producción y de interdependencia.

Por su parte, Chapa (2003) construye una matriz de contabilidad social de la economía mexicana para el año 2003 y, entre otras cosas, la utiliza para computar los multiplicadores contables y su descomposición en efectos propios, abiertos y circulares, con el fin de identificar las actividades que son importantes generadoras de renta nacional, y la importancia relativa del sector externo. Núñez (2003) construye una matriz de contabilidad social de México para el año 1996 y, también entre otras cosas, la utiliza para calcular los multiplicadores contables y analizar el efecto de inyecciones exógenas sobre el sector agropecuario y transferencias directas a los hogares más pobres. Los resultados obtenidos sugieren que estas últimas pueden tener efectos multiplicadores y redistributivos más importantes que los que se obtendrían con una inyección equivalente en el sector agropecuario. Aunque más alejado de los propósitos de nuestro artículo, el trabajo de Blancas (2006) utiliza una matriz de contabilidad social de 1990 para desarrollar, a partir del enfoque de los multiplicadores contables, un análisis de relaciones interinstitucionales, análogo al análisis interindustrial.

La construcción de matrices de contabilidad social de nivel estatal es también escasa en nuestro país; uno de los trabajos que han emprendido esta tarea es el de Chapa et al. (2008), quienes construyen una matriz de contabilidad social del estado de Nuevo León para 2004, con la cual llevan también a cabo un análisis de multiplicadores contables de la estructura de Nuevo León, a partir de la identificación de las cuentas estratégicas, según su poder generador de ingreso. Por su parte, Domínguez (2008) utiliza esta matriz para analizar el impacto de dos programas estatales y uno federal sobre los hogares más pobres de Nuevo León, también mediante modelo de multiplicadores contables.

En lo que sigue, el artículo se organiza de este modo: la sección I presenta la selección de la economía rural estudiada e información general de la misma. La sección II detalla la elaboración de la MCS y la metodología utilizada. En la sección III se calcula la matriz de multiplicadores generalizados, se analizan los impactos exógenos de interés y se calculan los índices de Rasmussen. La sección final concluye con algunos comentarios finales. 


\section{SELECCIÓN DE LA ECONOMIA RURAL ESTUDIADA}

El ejido Los Lirios, ubicado en el municipio de Arteaga del estado de Coahuila, fue seleccionado para esta investigación con base en los siguientes criterios:

1) Tamaño de la comunidad. El ejido cuenta con una población de 579 habitantes, distribuidos en 140 hogares, tamaño que optimiza los recursos financieros y humanos de los que se dispuso para el levantamiento de las encuestas necesarias para elaborar la matriz.

2) Diversidad de las fuentes de ingreso. Las actividades de producción dentro del ejido van desde el cultivo de diversos productos agrícolas, hasta la prestación de servicios. Esto permite analizar mejor las relaciones económicas dentro del ejido, dada la existencia de un mayor número de encadenamientos productivos.

3) Fuerte relación con el exterior. La comunidad mantiene relaciones con el exterior que son determinantes en la vida económica del ejido, tales como remesas, ingresos por ventas de productos en la región y transferencias gubernamentales. Estas relaciones permiten generar escenarios diversos a partir de cambios en las actividades productivas y/o de política económica.

Lo anterior permite asegurar que este estudio de caso proporcionará elementos que permitan comprender la estructura del sector rural en México, y en particular la estructura de comunidades como Los Lirios. Por otra parte, la aplicación de la metodología de Yúnez-Naude y Taylor (1999) permite elaborar una base de datos, que además de dar cuenta de la estructura de dicha economía, también posibilita el análisis de la forma en que distintos impactos afectarían la economía de la comunidad, de tal modo que los resultados pueden ser de utilidad como elementos de juicio para el diseño y/o instrumentación de políticas dirigidas a solventar problemáticas del sector rural.

El ejido Los Lirios, en la región sureste del estado de Coahuila, a 40 kilómetros de la cabecera municipal (Arteaga), dentro de la denominada Sierra de Arteaga, forma parte de la Sierra Madre Oriental, y se ubica a una altura de 2,340 metros sobre el nivel del mar.

Debido al tipo de clima existente en la zona geográfica donde se encuentra localizado el ejido, la agricultura es de riego. En este tipo de agricultura, el agua para el riego se obtiene principalmente de ríos, lagos, presas o pozos. Cabe hacer notar que en México sólo 30\% de las tierras cultivadas tienen agricultura de 
riego. En ella se emplean más recursos técnicos, menos mano de obra y su rendimiento es mayor.

Como dijimos, la comunidad de Los Lirios cuenta con una población total de 597 habitantes, de los cuales 350 son hombres y 247 son mujeres. La población infantil menor de 6 años representa $15.07 \%$ del total de la población, la población de entre 6 y 14 años representa $19.77 \%$ de la población total del ejido, es decir, 118 habitantes dentro de este rango, mientras que la población de 15 años y más representa $65.16 \%$ de la población con 389 habitantes. El promedio de edad es de 29 años.

El porcentaje de población que trabaja es de $36.01 \%$ del total. De los 215 habitantes que trabajan, 19 reciben menos de un salario mínimo mensual por su trabajo. La principal actividad económica dentro del ejido es la agricultura, además del comercio y la prestación de servicios. La actividad agrícola se encuentra muy diversificada, encontrándose cosecha de manzana y cultivos de chilacayote, frijol, maíz, papa, tomatillo y avena. Existe también actividad pecuaria y avícola, fundamentalmente de traspatio. Dentro de los negocios locales existe la comercialización de productos elaborados por los habitantes del ejido, como empanadas, vinos y dulces, todos elaborados con manzana.

La población alfabetizada representa $80.90 \%$ del total, es decir, 483 personas. Ésta se divide en 108 habitantes de entre 6 y 14 años y 375 habitantes de 15 años y mayores. La escolaridad promedio dentro del ejido es de 5.9 años.

\section{MCS PARA EL EJIDO LOS LIRIOS (MCSP-LL05)}

Este trabajo muestra la forma de elaborar una matriz de contabilidad social aplicada a pueblos (MCSP) con base en la metodología propuesta por Yúnez-Naude y Taylor (1999) para la elaboración de las MCs en zonas rurales, en donde la base de datos se obtuvo mediante encuestas especialmente diseñadas que se aplicaron en la zona rural elegida. Esta metodología representa una alternativa económica de acopio de información cuando se desea estudiar una economía a esta escala.

Es decir, el presente trabajo es una investigación empírica, que parte de una metodología ya existente, para la cual solamente se adecuan los instrumentos de recopilación de información, en este caso las encuestas aplicadas en Los Lirios.

Debido a la importancia del cuestionario para levantar la información y elaborar la MCs, y como es la parte de la metodología base que se modifica para este trabajo, hacemos aquí una exposición más detallada del mismo. Después de las modificaciones que se realizaron al cuestionario presentado por Yúnez-Naude y Taylor (1999), quedó dividido en 7 partes como sigue: 
- Hoja de control. Datos de quién, cuándo y cómo aplicó el cuestionario.

- El hogar. Se toma la definición propuesta por el INEGI, 2006: unidad formada por una o más personas que comparten un mismo gasto para alimentación y que residen habitualmente en una vivienda. Aquí se capturan datos que sirven de control para las demás secciones: nombre de los miembros del hogar, relación con el jefe del hogar, ocupación de cada quien, edad, sexo, edad en que comenzó a trabajar. Aunque estos datos estrictamente no son necesarios para elaborar la MCS.

- Migración. La información que se obtiene en esta sección sirve para conocer los ingresos provenientes del resto del país y del resto del mundo.

- Trabajo asalariado local y regional. En esta sección se obtiene información sobre la oferta y demanda de trabajo dentro de la comunidad y en el resto de la región: tipos de trabajo, salarios que se pagan, costos en que incurren los trabajadores, impuestos y ahorro de los asalariados, origen y destino de ingresos y costos.

- Actividades económicas. Aquí se registran las actividades productivas realizadas en el ejido, que pueden ser agrícolas, ganaderas, comerciales y de servicios. Ésta es una sección fundamental para elaborar la MCs, pues con esta información se construye la matriz de insumo-producto, que a su vez es la base para la MCS. Esta sección se subdivide en 9 apartados: $i$ ) tenencia de la tierra; ii) actividades agrícolas; cultivos básicos; iii) actividades agrícolas: cultivos perennes; $i v$ ) actividades pecuarias: ganado y aves; $v$ ) actividades pecuarias: producción de origen animal; vi) actividades agropecuarias: producción de solar; vii) actividades agropecuarias: recolección (leña, caza y pesca); viii) otras actividades no agropecuarias, y $i x$ ) actividades comerciales y de servicios.

- Otros ingresos y ahorros. Aquí se recaban datos sobre los ingresos de los hogares no generados por la actividad económica de los mismos, por ejemplo, transferencias y regalos entre hogares, préstamos, subsidios.

- Gastos del hogar. En esta sección se contabilizan los gastos de los hogares que no son parte de la actividad productiva: electricidad, agua, otros servicios, compra de bienes duraderos y no duraderos, medicinas y médicos, festividades.

La matriz de contabilidad social aplicada a pueblos (MCSP) es un instrumento analítico que registra las relaciones económicas que se dan dentro de las economías de los pueblos rurales y con el resto del mundo. Una característica fundamental de estas matrices es que pueden registrar relaciones económicas no monetizadas, como el autoconsumo, las transferencias en especie entre las vi- 
viendas, el uso del trabajo familiar, las remesas y las actividades de traspatio, ente otras.

El año de estudio elegido fue 2005, por dos motivos principales: a) por ser el año anterior más próximo al de la realización de las encuestas, con lo cual, las personas encuestadas tendrían una referencia más aproximada de los datos a recabar, y $b$ ) durante el año 2005 no se observaron sucesos extraordinarios que hubieran podido afectar el usual desempeño de las actividades socioeconómicas, por lo cual se puede considerar un periodo característico.

Para calcular el número de hogares a encuestar, se utilizaron la media y la varianza del ingreso de la población rural de México, obtenidas con base en datos de la Encuesta de Ingreso-Gasto de los Hogares (ENIGH, 2004) del INEGI.

Con los datos de la ENIGH-2004, y conociendo el número total de hogares en la población $(N)$, se obtuvo la cantidad de hogares a encuestar $(n)$. Tomando en cuenta que el ingreso rural nacional (INEGI, 2004) es de $\$ 72,106,505.00$, con una población total rural de 5,924,067 hogares, la media poblacional del ingreso rural nacional $(\mu)$, para una comunidad de 100 habitantes es de $\$ 1,217.17$.

$$
\mu=\frac{\$ 72,106,505.00}{5,924,067} \cdot 100=\$ 1,217.17
$$

Si se considera $95 \%$ de confiabilidad, la media muestral del ingreso de una comunidad se encuentra en un intervalo de \pm 12.5 en torno a la media del ingreso poblacional. Este intervalo expresa el grado de error y se denota con la letra $\varepsilon$, equivalente en este caso a $\$ 304.29$.

$$
\varepsilon=(\mu)(2 \cdot 0.125)=\$ 1,217.17 \cdot 0.25=\$ 304.29 .
$$

Para determinar el tamaño de la muestra con una distribución normal, se calcula su desviación estándar $(\sigma)$, la cual es igual al cociente del error permitido $(\varepsilon=\$ 304.29)$ entre el valor de la abscisa con un nivel de confianza de $80 \%$, el cual es comúnmente aceptado (Yúnez-Naude y Taylor, 1999); este valor es: $Z_{\alpha / 2}=0.3668$.

$$
\sigma=\frac{304.29}{0.3668}=829.59 \text {. }
$$

El valor de la desviación estándar es igual a $\$ 829.58$. Esto nos da una varianza poblacional igual a $\$ 688,224.82$. El tamaño de muestra para estimar el total poblacional está dado por:

$$
n=\frac{n_{o}}{1+\frac{n_{0}}{N}} \text { donde: } n_{o}=\frac{\sigma^{2} Z_{\alpha / 2}^{2}}{\varepsilon^{2}} .
$$


Sustituyendo los valores obtenidos:

$$
\begin{aligned}
\varepsilon & =\$ 304.29 \\
\sigma^{2} & =\$ 688,224.82 \\
Z_{\alpha / 2} & =1.96 \text { (con un nivel de confianza de } 95 \%) \\
N & =140 \text { total hogares ejido Los Lirios. }
\end{aligned}
$$

El resultado del cálculo realizado para estimar el tamaño de la muestra ( $n)$ en el caso de Los Lirios, es de 23.71, que se redondea a 24 hogares.

Una recomendación de Yúnez-Naude y Taylor (1999) es que si los recursos lo permiten se amplíe la muestra o incluso se haga un censo; en nuestro caso se optó por aumentar la muestra poblacional a 40 hogares.

Después de fijar el tamaño de la muestra, se procedió a seleccionar los hogares a encuestar, a partir de una tabla de números aleatorios (Green, 1999). Con la información de la tarjeta censal familiar, elaborada por la Secretaría de Salud para cada hogar, y proporcionada por el centro de salud de la comunidad, se obtuvieron los números de registro de cada hogar.

El siguiente paso fue seleccionar los hogares, dependiendo de los primeros 40 número distintos (tamaño de la muestra) que aparecen en la primera columna de la tabla de números aleatorios.

Para los casos en que no fue posible aplicar el cuestionario en alguno de los hogares seleccionados (por ejemplo, porque no se encontraba nadie en ese momento), se llevó a cabo el reemplazo utilizando el número de la tabla siguiente al último seleccionado.

\section{Definición del nivel de desagregación de la MCSP-LLO5}

De acuerdo con los objetivos de la investigación y con la información recabada por las encuestas, se determinó el nivel de desagregación de la MCSP-LL05, por lo cual, se realizó un nivel de desagregación exhaustivo de las actividades productivas, y en especial de las actividades agrícolas. Las cuentas de la MCSP-LL05 son las siguientes:

Actividades de producción: manzana, chilacayote, frijol, maíz, papa, tomatillo, avena forrajera, ganado y comercio. Factores de producción: tierra, capital, trabajo asalariado y trabajo familiar. Instituciones: hogares ejidales, hogares libres y gobierno. Capital: ahorro físico y ahorro humano. Exterior: resto de la 
región, resto del país y resto del mundo. "Hogares libres" se refiere a los hogares no ejidales, "ahorro físico" al ahorro que llevan a cabo los hogares como inversión física y "ahorro humano" se refiere a los gastos en educación.

\section{Los factores de expansión}

Debido a que la información recabada por medio de la encuesta sólo refleja las características del grupo de hogares que fueron seleccionados en la muestra representativa, y que para la construcción de la MCSP-LL05 se requieren datos de todo el pueblo, es necesario expandir los datos por medio de factores de expansión (FE).

Yúnez-Naude y Taylor (1999) muestran una forma sencilla de calcular los FE, que consiste en dividir el número total de hogares en el pueblo entre el número de hogares encuestados. El resultado obtenido se multiplica por cada uno de los datos que se requieren para elaborar la MCSP-LL05. Adicionalmente, se recomienda calcular los FE en forma desglosada, es decir, para cada tipo de hogar $\mathrm{y}$ de actividad productiva.

Para fines de este estudio, los hogares se dividieron en dos: hogares ejidales, y hogares libres. Los hogares ejidales (HE) se refieren a los hogares que poseen tierras dentro de la comunidad, mientras que el resto de los hogares son los llamados hogares libres (HL).

Por tanto, se calcularon FE para cada tipo de hogar, además de un FE para los comercios pertenecientes a la comunidad.

Partiendo del supuesto de que todos los ejidatarios produjeron en el año de estudio, el FE de los HE sirve para poder expandir los datos de producción agrícola del ejido.

Los cálculos de FE para Los Lirios son los siguientes:

$$
\begin{gathered}
f e . \text { de } h l=85(\text { total } h l) / 25(h l \text { encuestados })=3.4 . \\
f e . \text { de } h e=55(\text { total } h e) / 15(h e \text { encuestados })=3.67 .
\end{gathered}
$$

$f e$ de comercios $=11($ total comercios $) / 7($ comercios encuestados $)=1.57$.

Estos resultados fueron multiplicados por cada una de las cifras recabadas en los cuadros de control de datos, para posteriormente llenar las celdas de la MCSP-LL05. 


\section{Balance de la MCSP-LLO5}

Después de llenar todas las celdas de la MCSP-LL05, se deben sumar sus columnas y sus filas. Cabe recordar que una de las características de las MCs es que el total de sumas de filas de cada una de las cuentas debe ser igual a la suma de sus respectivas columnas.

Una vez terminado el llenado de las celdas de la MCSP-LL05, y al realizar la suma de las filas y las columnas, es común que ésta no esté balanceada. La técnica utilizada para el balance de la MCSP-LL05 consistió en modificar progresivamente los montos asignados a las cuentas exógenas. Es decir, no hay una cuenta residual propiamente dicha, sino que todas las cuentas se llenan con la información recabada, y luego se realiza un ajuste matemático que consiste esencialmente en hacer pequeñas modificaciones progresivas en las cuentas exógenas hasta que la matriz queda balanceada. En el párrafo siguiente se ofrece un ejemplo de este procedimiento.

Un ejemplo es el de la cuenta de gobierno, pues es improbable que el monto de lo que se recauda en impuestos dentro del ejido sea igual al que se reparte en el mismo en forma de subsidios. Por tanto, el déficit existente se compensa con la entrada de impuestos al gobierno del sector externo (en este caso, el resto de la región).

\section{Descripción de la MCSP-LL05}

Los datos de las celdas que conforman la MCSP-LL05 son los datos expandidos y revisados que se explicaron en las secciones anteriores. Estos datos se conjuntaron de acuerdo con la desagregación establecida anteriormente.

La MCSP-LL05 muestra las relaciones de los sectores económicos internos del ejido, así como las relaciones que tiene con los sectores del exterior. Estos sectores están conectados con otros, ya sea proveyendo los bienes y servicios que éstos demanden, o requiriendo insumos para la producción.

El cuadro 1 muestra la MCSP-LL05; realizando la descripción de la primer columna de la matriz aparece la producción de manzana, la cual pertenece a las actividades de producción; esta actividad tiene una producción total de $\$ 1,115,089.91$.

Para lograr esta producción, la actividad registra $\$ 168,266.42$ como gasto estimado del uso de la tierra utilizada, utiliza $\$ 256,362.39$ por concepto de compras de maquinaria y equipo, paga salarios por $\$ 18,577.75$ y el trabajo familiar 


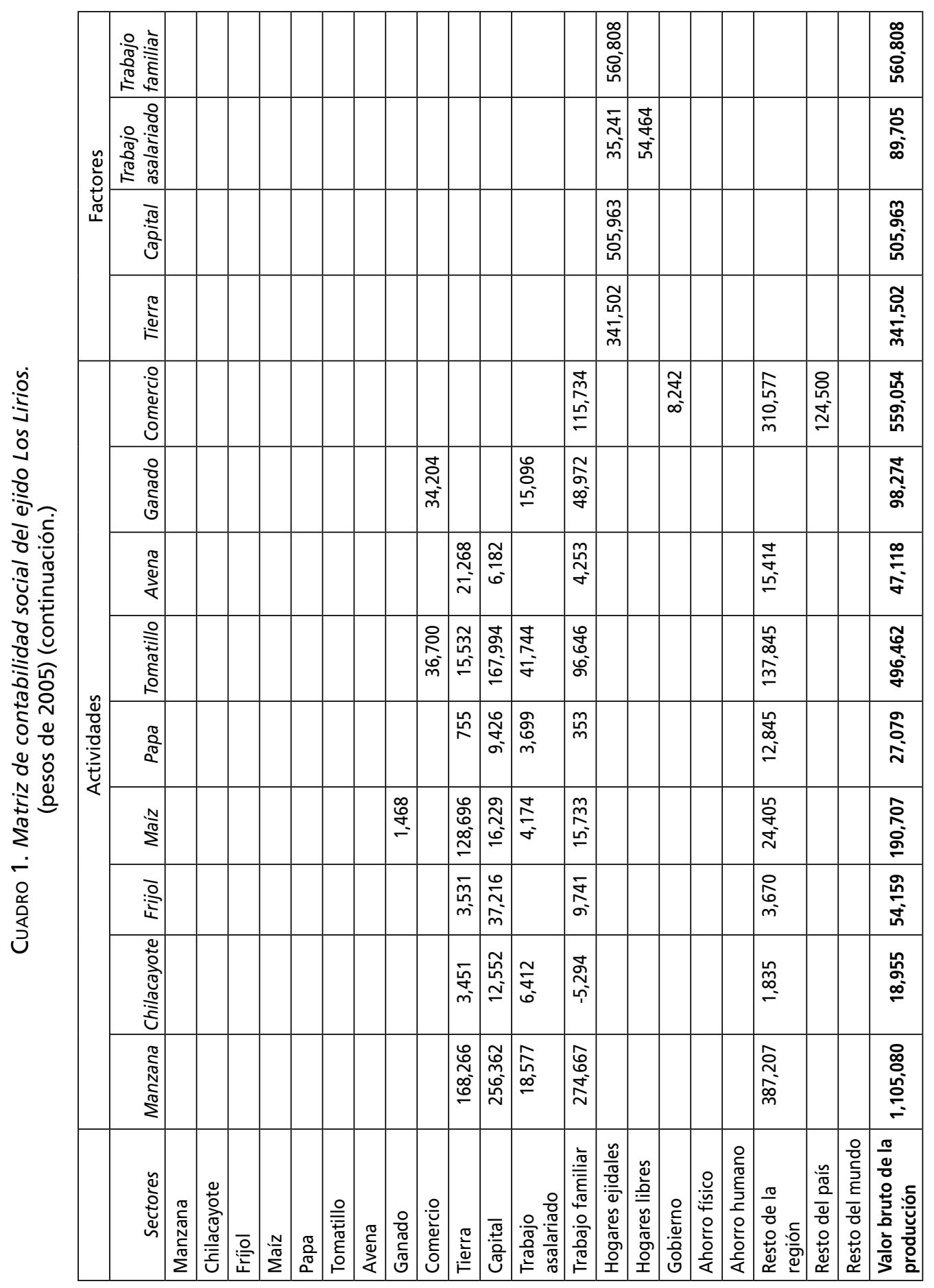




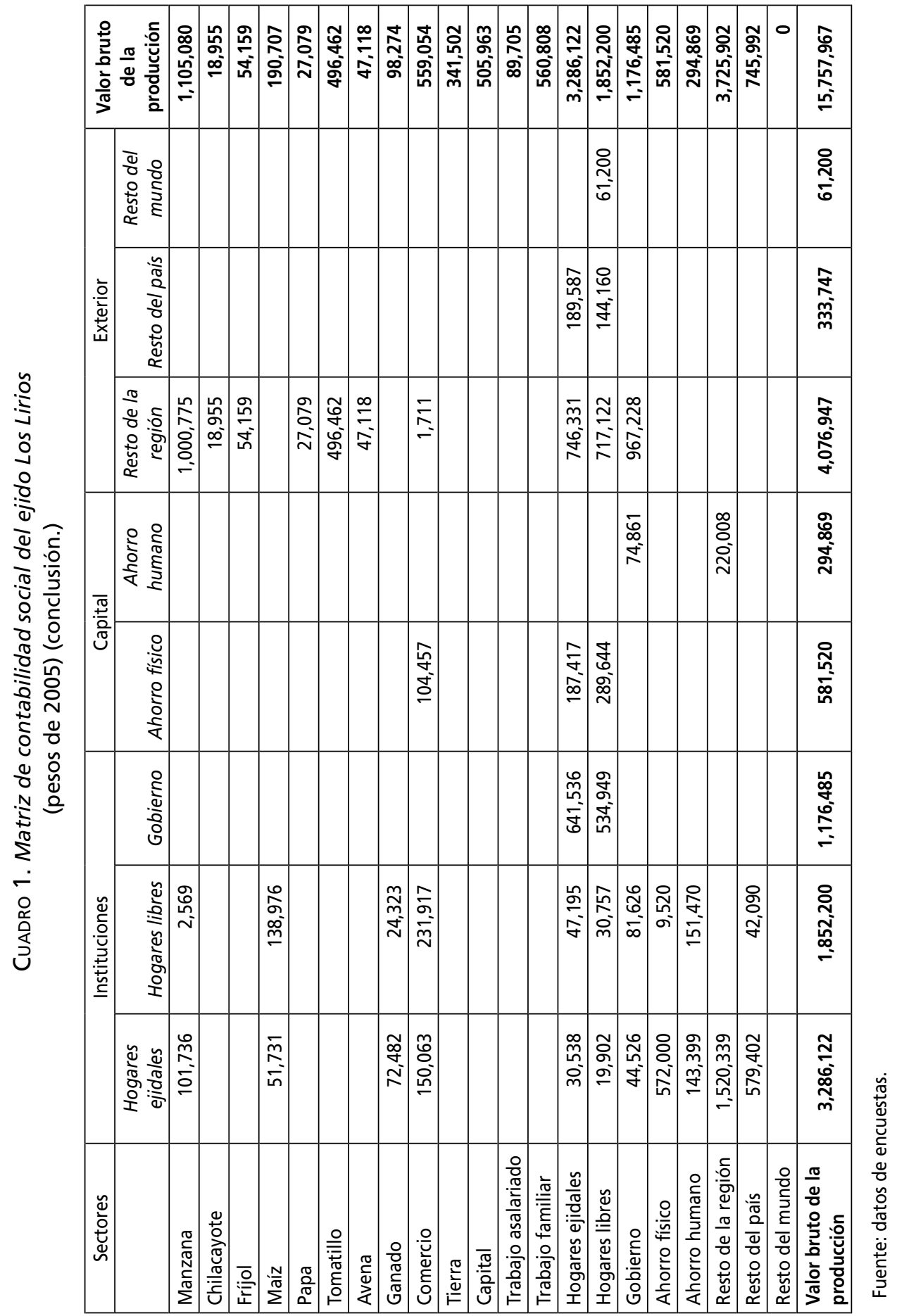


o ganancia tiene un total de $\$ 274,667.32$; por último, este sector utiliza insumos que obtiene del exterior por un total de $\$ 387,207.02$.

\section{MultiPliCAdORES GENERALIZAdOS, INYECCIONES EXóGENAS E ÍNDICES DE RASMuSSEN}

En esta sección primero se expone, resumidamente, el modelo teórico para la obtención de la matriz de multiplicadores generalizados, tomando como base de datos una MCSP, así como los índices de Rasmussen empleados para clasificar los sectores de la economía bajo estudio. En segundo lugar, se calculan y se analizan las matrices obtenidas y los índices de Rasmussen antes mencionados. Finalmente se estudian los impactos potenciales que algunas inyecciones exógenas seleccionadas podrían tener sobre la economía del ejido Los Lirios.

\section{El modelo de multiplicadores generalizados}

Los modelos de multiplicadores, con base tanto en una MCS o, como en este caso, en una MCSP, pueden concebirse como una generalización de los tradicionales modelos de insumo-producto de Leontief.

Para que la estructura contable de una MCSP resulte operativa para un modelo de multiplicadores, es necesario separar el conjunto de cuentas que conforman la matriz en cuentas endógenas y cuentas exógenas.

Las cuentas exógenas recogen instrumentos de política o variables que se determinan fuera del sistema económico estudiado. Generalmente, estas cuentas son las del gobierno (variación en transferencias) y el resto del mundo (variaciones en importaciones o exportaciones). Por otro lado, las cuentas endógenas son las cuentas de actividades de producción, factores de producción, hogares y empresas, cuyas variaciones se determinan internamente. En esta primera aproximación seguimos dicha clasificación para analizar las posibles consecuencias de inyecciones seleccionadas que podrían impactar en la economía de Los Lirios.

Cabe mencionar que el mayor detalle institucional que muestra la MCsP y el hecho de que en la parte endógena del modelo se incorporen no sólo las ramas de actividad sino también las rentas de los factores productivos y de los sectores institucionales endógenos, permite que los modelos MCs puedan captar un mayor número de intervenciones que los tradicionales modelos de insumo-producto (Cardenete, 2000, p. 19).

Teniendo en cuenta que en la MCSP el total de ingresos debe ser igual al total de gastos para cada una de sus $n$ cuentas, entonces: 


$$
Y_{i}=\sum_{j=1}^{n} Y_{i j}=\sum_{j=i}^{n} Y_{j i} \quad i, j=1,2, \ldots n,
$$

donde $Y_{i}$ es la renta total de la cuenta $i, Y_{i j}$ es el ingreso de la cuenta $i$ obtenido de la cuenta $j$, y $Y_{j i}$ son los gastos del sector $i$ en el sector $j$.

Por otra parte, podemos definir la matriz de coeficientes de gasto como:

$$
A_{n n}=\left(a_{i j}\right)=\frac{Y_{i j}}{Y_{j}}
$$

en donde los elementos $\left(a_{i j}\right)$ representan la proporción de las compras de la cuenta $j$ a la cuenta $i$, en el gasto total de la cuenta $j$.

Si definimos $m$ cuentas endógenas y $k$ cuentas exógenas con $n=m+k$, y sustituimos (2) en (1) obtenemos:

$$
Y_{i}=\sum_{j=1}^{n}\left(Y_{i j} / Y_{j}\right) Y_{j}=\sum_{j=1}^{n} a_{i j} Y_{j}=\sum_{j=1}^{m} a_{i j} Y_{j}+\sum_{j=m+1}^{n} a_{i j} Y_{j},
$$

donde la primera suma recoge el impacto sobre la cuenta $i$, de las rentas de las instituciones endógenas, y la segunda, el de las instituciones exógenas. Matricialmente, el sistema de ecuaciones (para las instituciones endógenas) puede escribirse como:

$$
y_{m}=A_{m m} y_{m}+A_{m k} y_{k}
$$

donde $y_{m}$ y $y_{k}$ son los vectores (columna) de renta de las instituciones endógenas y exógenas respectivamente, $A_{m m}$ y $A_{m k}$ las submatrices de orden $m \mathrm{x} m$ y $m \mathrm{x} k$ respectivamente, obtenidas al particionar la matriz de coeficientes de gasto $A_{n n}$ de acuerdo con la clasificación de instituciones endógenas y exógenas adoptada, como sigue:

$$
[A]=\left[a_{i j}\right]=\left[\begin{array}{cc}
A_{m m} & A_{m k} \\
A_{k m} & A_{k k}
\end{array}\right] \text {. }
$$

Si denotamos por $x_{m=}\left[A_{m k} y_{k}\right]$ al vector (columna) de rentas exógenas dirigidas a cada una de las instituciones endógenas, obtenemos inmediatamente:

$$
y_{m}=\left(I-A_{m m}\right)^{-1} x_{m}=M x_{m},
$$


donde $M$ es la matriz de multiplicadores generalizados (MMG). (Cuando las instituciones endógenas incluyen exclusivamente las actividades productivas, $M$ es la inversa de Leontief.) La matriz $M$ tiene la interpretación habitual: los elementos de la columna $j$ representan el impacto de un aumento de una unidad en la renta de la cuenta $j$ sobre las rentas de cada una de las instituciones endógenas, y los elementos en la diagonal principal son mayores o iguales que 1 .

\section{Definición de las cuentas exógenas}

Como uno de los objetivos de esta investigación es analizar posibles cambios en la demanda externa final y sus efectos en la economía de Los Lirios, así como evaluar el potencial efecto de determinadas inyecciones exógenas por parte del gobierno hacia la comunidad, definimos como cuentas exógenas las representadas por el gobierno y por el resto del mundo, el resto de las cuentas de la MCSPLL05 quedan como cuentas endógenas, cuyas rentas serán afectadas por las inyecciones de interés que analizaremos.

Dada esta clasificación, y después de analizar la MMG, llevaremos a cabo el análisis de tres principales inyecciones exógenas y de sus potenciales efectos en la economía bajo análisis: primero supondremos un aumento unitario en la demanda final del resto del mundo, y luego supondremos que el gobierno realiza una transferencia unitaria a los hogares de Los Lirios.

\section{Limitaciones del modelo de multiplicadores generalizados}

Es importante mantener presente que el análisis de los efectos de un impacto exógeno a partir de la MMG presenta limitaciones. Tal vez la más importante derive del hecho de que éste es un modelo de precios fijos, lo cual implica que un cambio en el precio de los bienes se trasladaría completamente hacia delante por completo, lo cual a su vez implica que el precio de los factores primarios es fijo. Ésta y otras limitaciones del modelo de multiplicadores se superan con el diseño e implantación de modelos de equilibrio general aplicado. Un excelente tratamiento sobre las propiedades e interpretación de los multiplicadores contables y de precios fijos se encuentra en Pyatt y Round (1979).

\section{4. Índices de Rasmussen}

A partir de la MMG también se calculan los índices de Rasmussen (1956), los cuales comparan los valores medios de los elementos de las columnas y filas de la 
MMG con el valor medio de todas las filas y columnas. Algebraicamente, el índice de arrastre o impacto (IA) de cada cuenta $j$ se define como la media de los elementos de la columna $j$, dividida por la media de las medias de todas las columnas, algebraicamente:

$$
U_{. j}=\frac{\frac{1}{m} \sum_{i=1}^{m} m_{i j}}{\frac{1}{m^{2}} \sum_{i=1}^{m} \sum_{j=1}^{m} m_{i j}}=\frac{\bar{m}_{. j}}{\frac{1}{m} \sum_{j=1}^{m} \bar{m}_{. j}} .
$$

Análogamente, el índice de dispersión (ID) de cada cuenta $i$ se define como la media de los elementos de la fila $i$ dividida por la media de las medias de todas las filas:

$$
U_{i .}=\frac{\frac{1}{m} \sum_{j=1}^{m} m_{i j}}{\frac{1}{m^{2}} \sum_{i=1}^{m} \sum_{j=1}^{m} m_{i j}}=\frac{\bar{m}_{i .}}{\frac{1}{m} \sum_{i=1}^{m} \bar{m}_{i .}}
$$

en donde $m m g=\left(m_{i j}\right)$, y $m$ es el número de cuentas endógenas.

Cuando el IA alcanza un valor superior a 1, se dice que esa cuenta es impulsora; cuando el ID tiene un valor superior a 1, la institución se considera estratégica, y cuando ambos son superiores a 1 se dice que la institución es clave para la economía. Aunque estos indicadores han sido criticados, permiten una útil primera aproximación a las interrelaciones productivas.

El cuadro 2 presenta la clasificación de las cuentas de la MCSP-LL05 según los índices de Rasmussen. De acuerdo con esto, la economía de Los Lirios tiene cuatro cuentas clave: la tierra, el capital, el trabajo familiar y el ahorro físico, lo cual nos llevaría a sugerir que, de llevarse a cabo programas de apoyo al sector rural, los recursos tendrían que enfocarse al fortalecimiento del capital social, es decir, del capital propiamente dicho, incluyendo la tierra, del trabajo familiar y de la inversión en capital humano.

Por otra parte, podemos observar que las cuentas con un índice de arrastre superior a la unidad son cuentas del sector agropecuario, mientras que aquellas con un índice de dispersión mayor que 1 , son las cuentas del comercio y de los hogares, lo cual indica que la estructura de esta economía es una en donde el sector agropecuario utiliza mayormente insumos del propio ejido, y que un incre- 
mento en la demanda de los mismos (a partir de programas de comercialización, por ejemplo) tendría importantes efectos multiplicativos sobre la economía del lugar. Aunque sería de interés realizar un análisis comparativo con base en estos indicadores, desafortunadamente hasta donde sabemos este tipo específico de análisis no se ha llevado a cabo para otros ejidos de México.

CUADRO 2. Índices de Rasmussen para la MCSP-LLO5

\begin{tabular}{|l|c|c|}
\hline & Índice de arrastre & Índice de dispersión \\
\hline Manzana & 1.067 & 0.524 \\
\hline Chilacayote & 1.325 & 0.376 \\
\hline Frijol & 1.368 & 0.376 \\
\hline Maíz & 1.305 & 0.558 \\
\hline Papa & 0.931 & 0.376 \\
\hline Tomatillo & 1.107 & 0.376 \\
\hline Avena & 1.092 & 0.376 \\
\hline Ganado & 1.272 & 0.503 \\
\hline Comercio & 0.596 & 1.191 \\
\hline Tierra & 1.065 & 1.117 \\
\hline Capital & 1.065 & 1.359 \\
\hline Trabajo asalariado & 1.029 & 0.684 \\
\hline Trabajo familiar & 1.065 & 1.124 \\
\hline Hogares ejidales & 0.689 & 4.714 \\
\hline Hogares libres & 0.631 & 1.443 \\
\hline Ahorro físico & 1.019 & 1.204 \\
\hline Ahorro humano & 0.376 & 0.700 \\
\hline
\end{tabular}

Fuente: datos de encuesta.

\section{Cálculo de la matriz de multiplicadores de la MCSP-LL05}

Puesto que el modelo de multiplicadores presupone que todas las cuentas están balanceadas, procedimos a modificar la MCSP-LL05 agregando las tres cuentas: resto de la región, resto del país y resto del mundo, en una sola cuenta balanceada. Luego se calculó la matriz de propensiones medias de gasto $\left(A_{n n}\right)$ que se presenta en el cuadro 3. La relevancia de esta matriz, en donde las primeras $m$ filas (columnas) representan las cuentas endógenas y las últimas $k$ filas (colum- 
nas) las cuentas exógenas, reside en que muestra el modo en que cada cuenta distribuye su gasto y, en particular, el efecto inmediato que un aumento en el gasto de una cuenta exógena tendrá sobre la renta de las cuentas exógenas (véase cuadro 3).

Con la submatriz $A_{m m}$, y resolviendo la fórmula (1.6), calculamos la MMG presentada en el cuadro 4. Como dijimos, cada columna muestra el efecto que tendría un aumento unitario exógeno (AUE) en el ingreso de esa cuenta, sobre la renta de las $M$ cuentas endógenas.

Por ejemplo, si la demanda por manzanas aumentase en una unidad, para satisfacer ese incremento la actividad encargada de producir manzanas tendría que aumentar su demanda de insumos en los montos dados por la columna manzanas de la matriz $A_{n n}$, como vimos antes. Luego, para satisfacer esa nueva demanda de insumos, las actividades implicadas tendrían que producir más, aumentando a su vez su demanda de insumos y así sucesivamente. Una vez que todos los efectos hayan tenido lugar, la actividad (columna) manzanas habrá incrementado su producción en $\$ 1.023$, el maíz en $\$ 0.018$, el ganado en $\$ 0.018$, el comercio en $\$ 0.074$, la tierra habrá incrementado su renta en $\$ 0.168$, el capital en $\$ 0.239$, el trabajo asalariado en $\$ 0.020$, el trabajo familiar en $\$ 0.28$, los hogares ejidales habrán subido su ingreso en $\$ 0.746$, los hogares libres en $\$ 0.083$, el ahorro físico en $\$ 0.13$ y el ahorro humano en $\$ 0.039$. La suma de la columna nos da el efecto multiplicador total, en este caso $\$ 2.838$. El mismo análisis vale para todas las demás cuentas endógenas.

La inspección de la MMG nos permite observar cuatro cuentas de la MMG con un efecto multiplicador superior a tres: frijol (\$3.64), chilacayote $(\$ 3.526)$, maíz (\$3.472), y ganado (\$3.384). Por otra parte, las cuentas con el menor efecto multiplicador son: hogares ejidales $(\$ 1.832)$, hogares libres $(\$ 1.678)$, comercio (\$1.586) y ahorro humano (\$1.00). Valga recordar que el ahorro humano se refiere a los gastos educativos, cuya totalidad se entrega al gobierno, por lo cual esa cuenta no tiene relación alguna con las cuentas endógenas.

\section{Aumentos unitarios exógenos}

Tal vez la principal aplicación de la MMG es que permite evaluar los efectos potenciales que cambios exógenos podrían tener sobre las cuentas endógenas del sistema. Ya vimos cómo las columnas de la MMG se pueden interpretar como el efecto de una inyección unitaria exógena sobre cada una de las cuentas endógenas, sin embargo, dicha inyección exógena puede ser distribuida entre las cuentas endógenas de muchos modos distintos. En este apartado analizamos tres dis- 


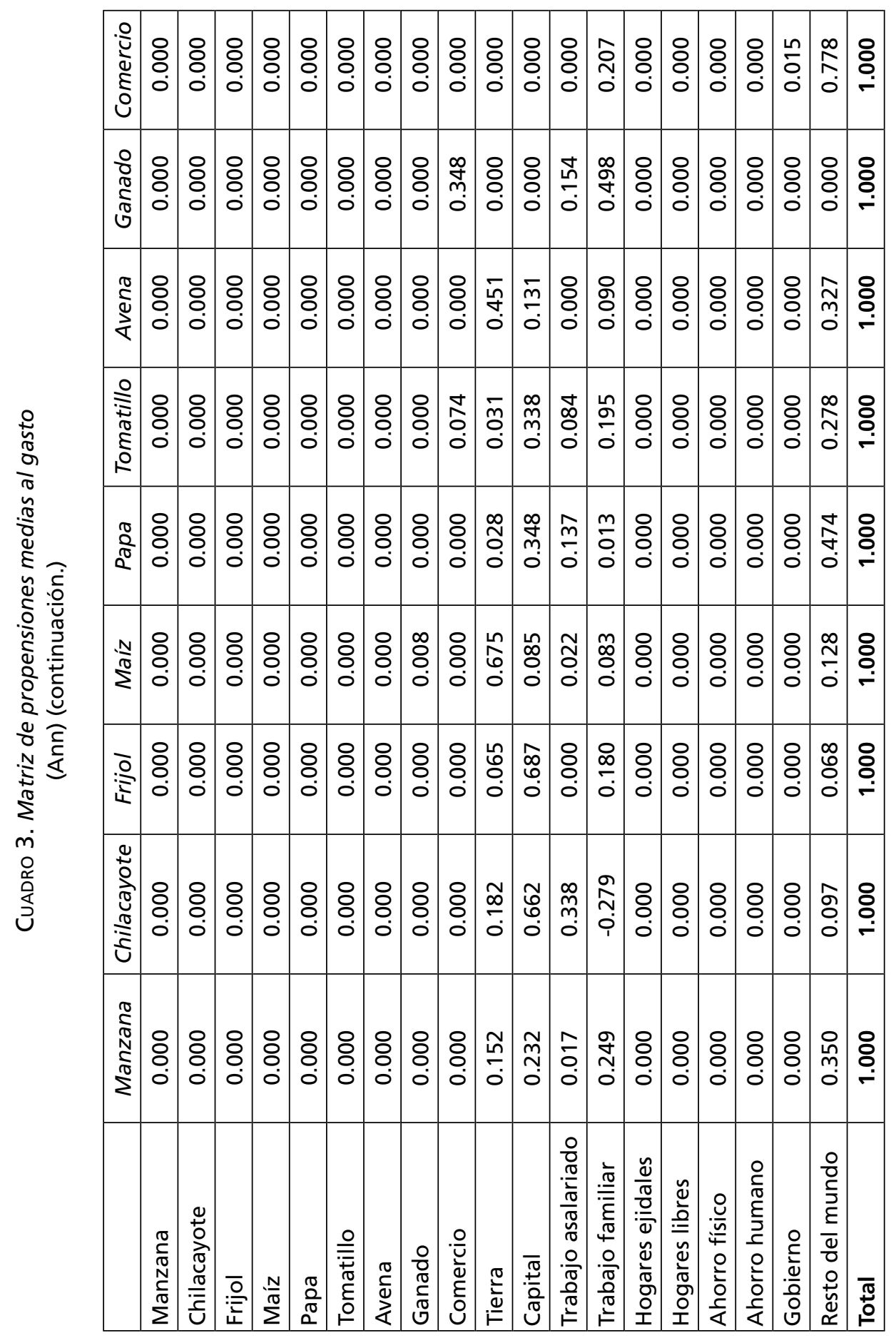




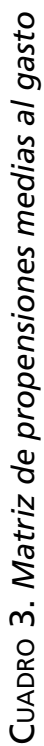

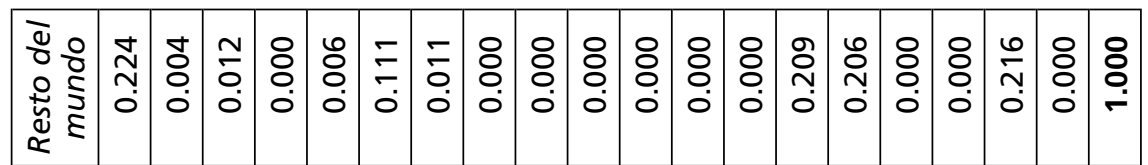

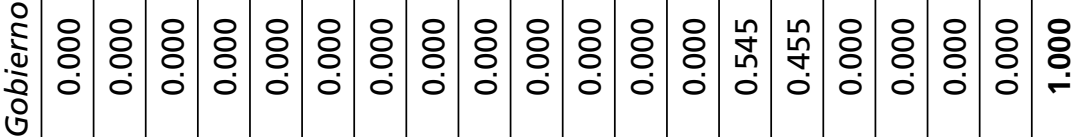

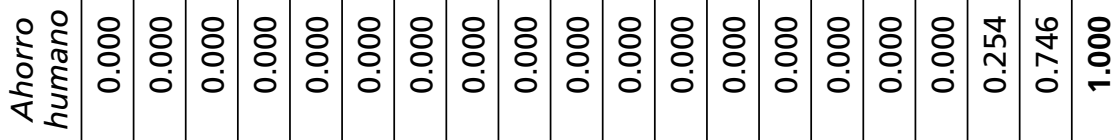

온

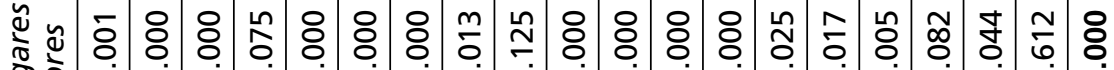

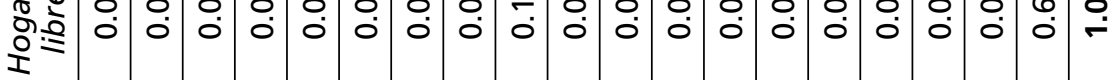

๘े

そ

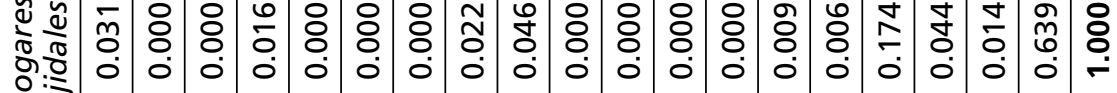

ㅇํㅇ 엉

造

ชิ

雨 장

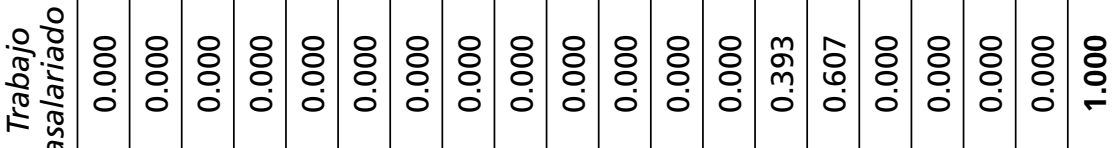

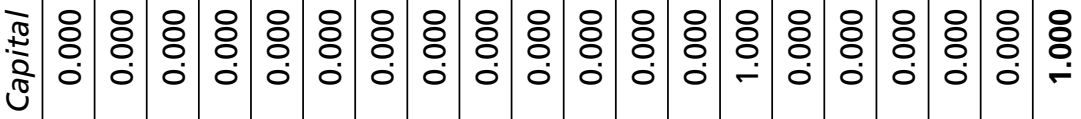

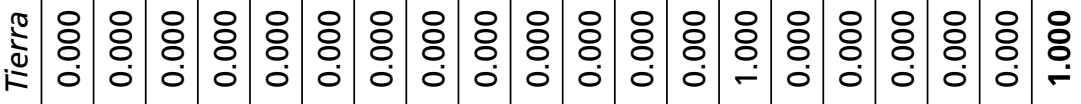

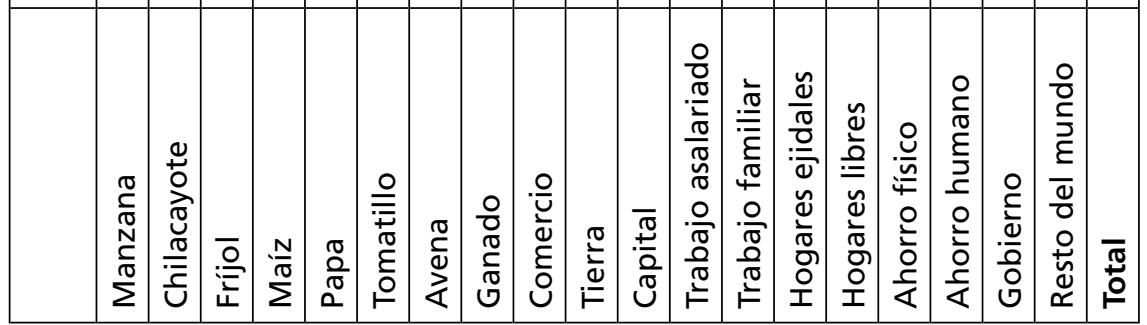




\begin{tabular}{|c|c|c|c|c|c|c|c|c|c|c|c|c|c|c|c|c|c|c|}
\hline 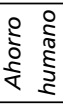 & & & & & & & & & & & & & & & & & $\stackrel{\text { ঃ }}{\circ}$ & $\stackrel{\text { ঃ }}{\circ}$ \\
\hline 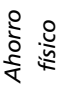 & \begin{tabular}{|l|}
0 \\
0 \\
0 \\
0
\end{tabular} & & & $\begin{array}{l}\text { 응 } \\
\text { O. }\end{array}$ & & & & $\frac{\sigma}{\circ}$ & $\begin{array}{l}\text { స్ } \\
\text { O }\end{array}$ & $\begin{array}{l}0 \\
\text { m} \\
0 \\
0\end{array}$ & $\begin{array}{l}\infty \\
0 \\
0 \\
0\end{array}$ & $\begin{array}{l}\text { ப் } \\
\text { Oे }\end{array}$ & $\begin{array}{l}\stackrel{\infty}{\circ} \\
0 \\
0\end{array}$ & 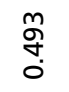 & $\begin{array}{l}\hat{n} \\
\text { ñ } \\
0\end{array}$ & $\begin{array}{l}\stackrel{\circ}{\circ} \\
\text { ọ } \\
-\end{array}$ & $\begin{array}{l}\hat{0} \\
0 \\
0\end{array}$ & $\underset{⿱ 亠}{N}$ \\
\hline 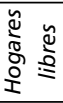 & $\begin{array}{l}0 \\
\\
0 \\
0\end{array}$ & & & $\begin{array}{l}\text { ㅇ } \\
\text { O. } \\
0\end{array}$ & & & & $\frac{\infty}{0}$ & $\frac{\stackrel{\infty}{+}}{0^{\circ}}$ & $\begin{array}{l}\text { Ln } \\
\text { กิำ } \\
0\end{array}$ & $\begin{array}{l}\infty \\
0 \\
0 \\
0\end{array}$ & 농 & \begin{tabular}{l}
$\infty$ \\
\multirow{0}{*}{} \\
0
\end{tabular} & $\frac{\bar{n}}{0}$ & $\stackrel{\hat{m}}{\circ}$ & $\underset{\tilde{O}}{\tilde{O}}$ & סั & $\stackrel{\infty}{\stackrel{\infty}{6}}$ \\
\hline 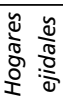 & \begin{tabular}{|l|} 
\\
0 \\
0 \\
0 \\
0 \\
\end{tabular} & & & స̃ & & & & $\begin{array}{l}\text { స } \\
\text { O }\end{array}$ & $\frac{m}{\frac{m}{\sigma}}$ & $\begin{array}{l}\text { Ð } \\
\text { ல }\end{array}$ & бo & 농 & \begin{tabular}{l}
$\infty$ \\
\multirow{0}{0}{} \\
0
\end{tabular} & $\stackrel{\Xi}{\check{-}}$ & $\frac{m}{\frac{m}{0}}$ & 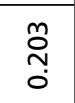 & $\begin{array}{l}8 \\
\varnothing \\
0\end{array}$ & $\underset{\mathscr{\infty}}{\stackrel{\infty}{-}}$ \\
\hline 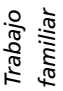 & \begin{tabular}{|c|}
0 \\
$\tilde{m}$ \\
0 \\
0 \\
\end{tabular} & & & స్ & & & & స్ & $\frac{m}{\frac{m}{0}}$ & $\begin{array}{l}\text { ¿ } \\
0 \\
0\end{array}$ & 후 & $\begin{array}{l}n \\
\stackrel{0}{0} \\
0\end{array}$ & 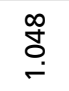 & $\underset{\square}{\stackrel{\Xi}{-}}$ & $\frac{m}{\frac{m}{0}}$ & 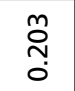 & $\begin{array}{l}8 \\
0 \\
0\end{array}$ & $\begin{array}{l}\underset{N}{\infty} \\
\infty \\
\text { N }\end{array}$ \\
\hline 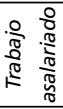 & \begin{tabular}{|l|}
$\infty$ \\
\\
0 \\
0
\end{tabular} & & & $\begin{array}{l}\text { 옹 } \\
\text { Oे }\end{array}$ & & & & స్ & $\stackrel{n}{m}$ & $\underset{\dot{O}}{\stackrel{m}{+}}$ & $\begin{array}{l}9 \\
8 \\
0\end{array}$ & $\stackrel{\text { 옹 }}{\circ}$ & $\begin{array}{l}\infty \\
\text { O } \\
0\end{array}$ & 守 & $\begin{array}{l}\mathbb{T} \\
6 \\
0\end{array}$ & 용 & $\begin{array}{l}\frac{9}{0} \\
0 \\
0\end{array}$ & 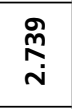 \\
\hline 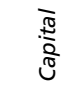 & $\mid$\begin{tabular}{l|}
0 \\
$\tilde{m}$ \\
0 \\
0 \\
\end{tabular} & & & 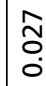 & & & & $\begin{array}{l}\text { I } \\
0 \\
0\end{array}$ & $\frac{m}{\frac{m}{\sigma}}$ & 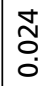 & бㅜ & $\begin{array}{l}\text { 웅 } \\
\text { ○े }\end{array}$ & $\begin{array}{l}\infty \\
\text { O̊ } \\
0\end{array}$ & 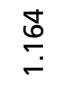 & $\frac{m}{\frac{m}{\sigma}}$ & 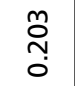 & $\begin{array}{l}\circ \\
\varnothing \\
0\end{array}$ & $\begin{array}{c}\underset{\infty}{\infty} \\
\text { N }\end{array}$ \\
\hline 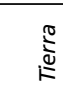 & $\left|\begin{array}{l}0 \\
\tilde{y} \\
0 \\
0\end{array}\right|$ & & & స్ & & & & $\begin{array}{l}\text { సે } \\
\text { O. }\end{array}$ & $\frac{m}{\frac{m}{\sigma}}$ & さ্ণ & 중 & $\begin{array}{l}\text { 농 } \\
\text { O̊ }\end{array}$ & \begin{tabular}{l}
$\infty$ \\
\multirow{O}{0}{} \\
0
\end{tabular} & 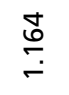 & $\frac{m}{\frac{m}{\sigma}}$ & 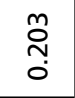 & \begin{tabular}{l}
$\circ$ \\
\hdashline \\
0
\end{tabular} & $\begin{array}{c}\underset{\infty}{\infty} \\
\stackrel{N}{N}\end{array}$ \\
\hline 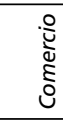 & $\begin{array}{l}0 \\
0 \\
0 \\
0\end{array}$ & & & $\begin{array}{l}\text { ठ } \\
\text { ठ }\end{array}$ & & & & $\begin{array}{l}\mathscr{0} \\
\circ \\
\dot{0}\end{array}$ & $\underset{\overbrace{}}{\overparen{O}}$ & 옹 & 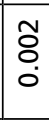 & ¿্口 & $\underset{\sim}{N}$ & 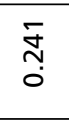 & 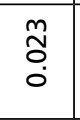 & 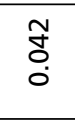 & $\stackrel{N}{0}$ & $\begin{array}{l}\stackrel{0}{0} \\
\stackrel{0}{n} \\
\end{array}$ \\
\hline \begin{tabular}{l}
$\frac{8}{8}$ \\
\multirow{0}{0}{} \\
0 \\
0
\end{tabular} & $\begin{array}{l}\tilde{\Xi} \\
0 \\
0\end{array}$ & & & $\begin{array}{l}\stackrel{0}{0} \\
0 \\
0\end{array}$ & & & & $\stackrel{\circ}{\circ}$ & 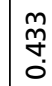 & 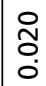 & $\begin{array}{l}\infty \\
0 \\
0 \\
0\end{array}$ & $\frac{n}{0}$ & $\begin{array}{l}\text { L } \\
\stackrel{0}{0} \\
0\end{array}$ & $\frac{\stackrel{\infty}{+}}{\vdots}$ & $\frac{\infty}{0}$ & $\frac{\bar{m}}{\dot{0}}$ & $\begin{array}{l}0 \\
\text { O̊ } \\
0\end{array}$ & 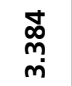 \\
\hline 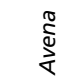 & \begin{tabular}{l}
\multirow{J}{0}{} \\
0 \\
0
\end{tabular} & & & $\frac{\infty}{0}$ & & & $\stackrel{8}{\circ}$ & $\frac{\infty}{0}$ & $\begin{array}{l}\stackrel{0}{0} \\
\stackrel{0}{0}\end{array}$ & $\begin{array}{l}\hat{\sigma} \\
0 \\
0\end{array}$ & $\begin{array}{l}\infty \\
\stackrel{m}{0} \\
0\end{array}$ & $\begin{array}{l}\text { ठ্ } \\
\text { ¿े }\end{array}$ & $\stackrel{\stackrel{m}{N}}{0}$ & 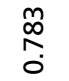 & 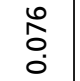 & $\frac{\hat{m}}{0}$ & $\begin{array}{l}\text { O্ণ } \\
\text { Oे }\end{array}$ & 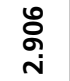 \\
\hline 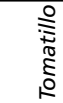 & $\underset{\Xi}{\tilde{O}}$ & & & $\begin{array}{l}\overline{0} \\
0 \\
0\end{array}$ & & $\stackrel{\circ}{\circ}$ & & $\stackrel{\infty}{\circ}$ & $\frac{\bar{n}}{\circ}$ & 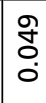 & 告 & $\begin{array}{l}\infty \\
0 \\
0 \\
0\end{array}$ & 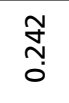 & 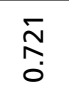 & $\frac{\underset{N}{0}}{0}$ & 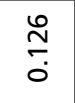 & $\begin{array}{l}\bar{\delta} \\
0\end{array}$ & ఫั \\
\hline$\frac{8}{8}$ & $\begin{array}{l}1 \\
0 \\
0\end{array}$ & & & $\frac{\sigma}{0}$ & $\stackrel{8}{\circ}$ & & & $\frac{\pi}{0}$ & $\begin{array}{l}\mathscr{0} \\
\ddot{0}\end{array}$ & 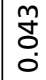 & $\begin{array}{l}\stackrel{+}{n} \\
\text { m. } \\
0\end{array}$ & $\frac{\stackrel{\sigma}{m}}{0}$ & $\begin{array}{l}\stackrel{\infty}{\infty} \\
0 \\
0\end{array}$ & $\begin{array}{l}\stackrel{\infty}{\hat{N}} \\
\text { م. }\end{array}$ & $\frac{\stackrel{o}{m}}{\dot{0}}$ & $\begin{array}{l}\text { m. } \\
\text { : } \\
0\end{array}$ & $\begin{array}{l}\text { rे } \\
\stackrel{0}{0}\end{array}$ & 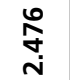 \\
\hline$\sum^{\frac{N}{\pi}}$ & $\begin{array}{l}\bar{m} \\
0 \\
0 \\
0\end{array}$ & & & 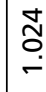 & & & & $\stackrel{\bar{m}}{0}$ & $\frac{\widetilde{\sigma}}{\circ}$ & ঢొ & ठั & 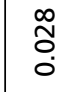 & $\frac{\stackrel{D}{~}}{0}$ & $\begin{array}{l}\text { م̆ } \\
\text { مุ }\end{array}$ & $\frac{\mathfrak{N}}{\check{0}}$ & $\frac{⿱}{\stackrel{D}{0}}$ & 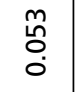 & 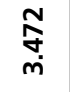 \\
\hline 产 & $\begin{array}{l}\tilde{m} \\
\text { ơ } \\
0\end{array}$ & & ¿ & 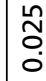 & & & & 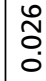 & ๕. & $\mid \begin{array}{l}\hat{\infty} \\
0 \\
0\end{array}$ & $\begin{array}{l}\hat{\sigma} \\
0 \\
0\end{array}$ & $\begin{array}{l}\text { LO } \\
\text { Oे }\end{array}$ & 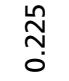 & $\begin{array}{l}L_{\infty}^{\infty} \\
\stackrel{0}{O}\end{array}$ & 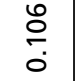 & $\frac{\infty}{\stackrel{\infty}{0}}$ & $\begin{array}{l}\text { L̊ } \\
0 \\
0\end{array}$ & $\begin{array}{l}\text { Q } \\
\text { mi }\end{array}$ \\
\hline స̃ & $\begin{array}{l}\hat{\vdots} \\
\vdots \\
0 \\
0\end{array}$ & ஓ̊ㅗ․ & & $\begin{array}{l}\stackrel{\operatorname{Ln}}{\tilde{O}} \\
\stackrel{0}{0}\end{array}$ & & & & $\begin{array}{l}\stackrel{\sim}{0} \\
0\end{array}$ & 읃 & $\stackrel{0}{N}$ & $\begin{array}{l}\pi \\
0 \\
0\end{array}$ & $\underset{m}{m}$ & $\begin{array}{l}\stackrel{\oplus}{N} \\
\stackrel{1}{i}\end{array}$ & 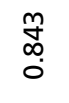 & ָָ & $\frac{\stackrel{\infty}{+}}{\dot{0}}$ & $\begin{array}{l}\overline{0} \\
0 \\
0\end{array}$ & $\begin{array}{l}\stackrel{N}{n} \\
\text { min }\end{array}$ \\
\hline $\begin{array}{l}\text { D̃ } \\
\mathbb{N} \\
\stackrel{2}{\pi} \\
\Sigma\end{array}$ & 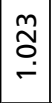 & & & $\frac{\infty}{0}$ & & & & $\stackrel{\infty}{\circ}$ & 站 & $\begin{array}{l}\infty \\
0 \\
0 \\
0\end{array}$ & 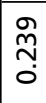 & $\begin{array}{l}\stackrel{O}{0} \\
\text { Oे }\end{array}$ & $\underset{\stackrel{O}{0}}{\stackrel{\infty}{0}}$ & $\begin{array}{l}\stackrel{0}{N} \\
\vdots \\
0\end{array}$ & $\begin{array}{l}m \\
0 \\
0 \\
0\end{array}$ & $\frac{\stackrel{O}{m}}{\dot{0}}$ & $\begin{array}{l}\text { ஸे } \\
\text { Oे }\end{array}$ & 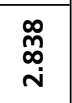 \\
\hline & $\begin{array}{l}\mathbb{0} \\
\stackrel{2}{\pi} \\
N \\
N \\
\frac{1}{0} \\
\Sigma\end{array}$ & 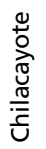 & 总 & $\frac{N}{N}$ & $\frac{\pi}{\sigma}$ & 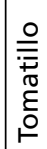 & 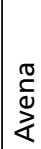 & $\begin{array}{l}\frac{0}{0} \\
\frac{0}{0} \\
\frac{1}{0} \\
0\end{array}$ & 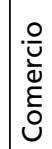 & $\stackrel{\frac{\pi}{2}}{=}$ & 京 & 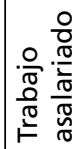 & 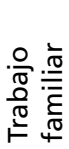 & 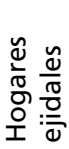 & 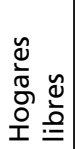 & $\begin{array}{l}0 \\
\frac{0}{2} \\
\frac{0}{4} \\
\frac{c}{4}\end{array}$ & 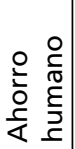 & 횰 \\
\hline
\end{tabular}


tintas formas de repartir el AUE entre las cuentas endógenas, que son de interés para el presente trabajo.

\section{Un aumento unitario exógeno homogéneamente distribuido}

Generalmente, para utilizarlo como punto de comparación, resulta de interés analizar los efectos que se observarían si el AUE se distribuye homogéneamente entre las cuentas endógenas, en este caso, los efectos quedan dados por la media de los elementos de las filas de la MMG.

En la columna "homogéneo" del cuadro 5 se presentan los resultados de dicha inyección. Como podemos observar, en este caso los más beneficiados serían los hogares ejidales con $\$ 0.738$, seguidos por los hogares libres con $\$ 0.226$, y el capital con $\$ 0.213$.

El efecto multiplicador total sería de \$2.661 (véase cuadro 5).

CUADRo 5. Aumentos unitarios exógenos seleccionados

\begin{tabular}{|l|c|c|c|c|}
\hline & Homogéneo & Gobierno & Agrícola & Resto del mundo \\
\hline Manzana & 0.082 & 0.023 & 0.150 & 0.308 \\
\hline Chilacayote & 0.059 & 0.000 & 0.125 & 0.005 \\
\hline Frijol & 0.059 & 0.000 & 0.125 & 0.015 \\
\hline Maíz & 0.087 & 0.051 & 0.148 & 0.037 \\
\hline Papa & 0.059 & 0.000 & 0.125 & 0.008 \\
\hline Tomatillo & 0.059 & 0.000 & 0.125 & 0.142 \\
\hline Avena & 0.059 & 0.000 & 0.125 & 0.013 \\
\hline Ganado & 0.079 & 0.023 & 0.146 & 0.020 \\
\hline Comercio & 0.186 & 0.129 & 0.139 & 0.116 \\
\hline Tierra & 0.175 & 0.038 & 0.217 & 0.085 \\
\hline Capital & 0.213 & 0.010 & 0.318 & 0.141 \\
\hline Trabajo asalariado & 0.107 & 0.005 & 0.098 & 0.024 \\
\hline Trabajo familiar & 0.176 & 0.048 & 0.176 & 0.144 \\
\hline Hogares ejidales & $\mathbf{0 . 7 3 8}$ & $\mathbf{0 . 7 0 3}$ & $\mathbf{0 . 8 0 7}$ & 0.702 \\
\hline Hogares libres & $\mathbf{0 . 2 2 6}$ & $\mathbf{0 . 5 3 3}$ & 0.137 & $\mathbf{0 . 3 5 0}$ \\
\hline Ahorro físico & 0.188 & 0.125 & 0.141 & 0.124 \\
\hline Ahorro humano & 0.109 & 0.074 & 0.046 & 0.059 \\
\hline Total & $\mathbf{2 . 6 6 1}$ & $\mathbf{1 . 7 6 2}$ & $\mathbf{3 . 1 4 9}$ & $\mathbf{2 . 2 9 3}$ \\
\hline
\end{tabular}

Fuente: $M M G$. 


\section{Una inyección gubernamental}

El interés de tratar de evaluar los posibles efectos de una transferencia gubernamental a una comunidad es casi obvio, y más aún en un país como México, en donde los grados de pobreza extrema han conducido a la implantación de programas de combate a la pobreza a partir de transferencias directas.

De acuerdo con la matriz de coeficientes de gasto de la MCSP-LL05, un incremento de una unidad en el gasto del gobierno redundaría en un incremento de $\$ 0.545$ en el ingreso de los hogares ejidales, y de $\$ 0.455$ en el ingreso de los hogares libres; en la columna "gobierno" del cuadro 5 podemos ver los efectos de esta inyección. Obviamente, los más beneficiados serían los hogares, que incrementarían su ingreso en $\$ 0.703$ y $\$ 0.533$ respectivamente, seguidos por el comercio con $\$ 0.129$, y por el ahorro físico con $\$ 0.125$. El efecto multiplicador total sería de $\$ 1.762$.

\section{Un aumento en la demanda del resto del mundo}

Analizamos la posibilidad de un incremento en la demanda del resto del mundo, el cual puede obedecer a diversas causas; aquí simplemente suponemos un aumento unitario en la demanda del resto del mundo, de acuerdo con las ponderaciones dadas por la columna "resto del mundo" en el cuadro 3 (estos coeficientes se normalizan para que sumen 1 cuando algunas de las entradas de la columna $k$ de la matriz $A_{k k}$ no son nulas). Los efectos de este incremento están en la columna "resto del mundo" del cuadro 5, en donde podemos notar que los sectores más beneficiados son los hogares ejidales y libres los cuales aumentarían respectivamente su ingreso en $\$ 0.702$ y $\$ 0.350$, además de la producción de manzana que incrementaría su ingreso en $\$ 0.308$. La variación total producida por este incremento sería de $\$ 2.293$.

\section{Un subsidio agrícola}

Un posible subsidio al sector agrícola es otra de las políticas de indudable interés por varias razones, la crisis del campo mexicano, las desgravaciones del TLCAN, los subsidios otorgados en otros países. Aquí supondremos que la inyección unitaria se distribuye homogéneamente entre las actividades agrícolas. La columna "agrícola" del cuadro 5 muestra los resultados. 


\section{Las remesas}

Por último, valga notar que de acuerdo con la MCSP-LL05, el "resto del mundo" solamente dirige transferencias a los hogares libres. Según esto, podemos apreciar los efectos de un incremento unitario en las remesas procedentes de Canadá - las remesas del resto del mundo para el caso de Los Lirios dependen del Programa de Trabajadores Agrícolas Temporales, conocido como SAWP, por sus siglas en inglés, el cual envía trabajadores a Canadá-, observando directamente la columna hogares libres de la MMG (cuadro 4). En dicha columna se ve que la transferencia tiene el mayor efecto sobre los hogares libres obviamente (1.037), seguido por los hogares ejidales (0.151) y por el comercio (0.148). El efecto multiplicador total es relativamente bajo (1.678).

Finalmente, es importante subrayar que en el análisis de las políticas que representan inyecciones exógenas, el modelo no toma en cuenta cómo está siendo financiada la política, es decir, se supone implícitamente que el financiamiento de las políticas no se lleva a cabo con recursos endógenos a la comunidad, como podría ser un impuesto sobre los hogares (o sobre las actividades productivas), sino que dichos recursos provendrían de fondos exógenos, posiblemente del gobierno federal o del resto del mundo.

\section{Comentarios finales}

La importancia de los estudios basados en matrices de contabilidad social consiste en que permiten conocer el perfil socioeconómico de comunidades pequeñas. Al construir la MCSP-LL05 se obtuvo información que ilustra claramente las características de la economía del ejido.

De acuerdo con los objetivos de este trabajo, se construyó la MCSP-LL05, la cual muestra una estructura económica muy dependiente de las relaciones económicas con el exterior. La razón de esta dependencia se encuentra en el lado de las fuentes de ingresos, a partir de las actividades que la mayoría de la población realiza como asalariada u ofreciendo servicios en localidades cercanas, además de que las ventas de las actividades agrícolas son sumamente dependientes del exterior. Por el lado del consumo, los ingresos percibidos por los miembros del hogar se emplean regionalmente, es decir, se gastan en gran medida en bienes de consumo e insumos provenientes de la zona metropolitana de Arteaga y Saltillo.

El presente estudio evidencia que aun una pequeña comunidad campesina como Los Lirios tiene una estructura socioeconómica compleja que debe ser considerada en el diseño de las políticas dirigidas a su mejoramiento. 
Por otro lado, los modelos de multiplicadores aplicados a poblaciones del México rural permiten estudiar los impactos que tienen y podrían tener en el agro y los ingresos de los hogares los cambios en la economía y en la política gubernamental de desarrollo.

En este trabajo se analizaron cuatro impactos exógenos por medio de la MMG, en donde se encontró que, en general, la aplicación de un subsidio repartido homogéneamente entre las actividades agrícolas tiene una mayor repercusión positiva en la economía del ejido; sin embargo, el efecto en el ingreso de los hogares del ejido es muy desigual, siendo los hogares ejidales los que obtienen mayores beneficios.

En el caso del impacto que plantea un incremento de los subsidios gubernamentales, lo cual aumentaría los ingresos de los dos tipos de hogares dentro de la comunidad, se puede observar que éste posee un poder de encadenamiento poco significativo sobre las actividades de producción del ejido debido a que la mayor parte del nuevo ingreso, generado por dicho subsidio, se gastaría en bienes provenientes del exterior.

Los impactos que proponen un incremento homogéneo sobre todas las cuentas endógenas de la MCSP-LL05, o un aumento de la demanda del "resto del mundo", arrojan resultados similares, obteniendo mayores beneficios los hogares ejidales respecto a los hogares libres.

Evidentemente, entre los distintos escenarios planteados, los hogares ejidales son los que presentan incrementos más significativos en su ingreso, debido a que estos hogares son los dueños de los factores de producción utilizados por las distintas actividades de producción pertenecientes al ejido.

Por otra parte, la MCSP-LL05 aquí detallada puede utilizarse para llevar a cabo análisis más refinados, ya sea a partir del diseño de modelos de equilibrio computable o aplicado, o de análisis estructurales más complejos.

\section{REFERENCIAS BIBLIOGRÁFICAS}

Adelman, I., E. Taylor, y S. Vogel (1987), "Life in a Mexican Village: A SAM Perspective", The Journal of Development Studies.

Barceínas, F., et al. (1997), "Multiplicadores contables y de precios fijos: Una aplicación a una matriz de contabilidad social para México (1989)", La crisis productiva y financiera mexicana, UAM-Azcapotzalco, México.

__ , y H. Cervini (1993), "Análisis de los multiplicadores contables asociados a una matriz de contabilidad social para México”, Análisis Económico, volumen 11, número 22. 
Blancas, A. (2006), "Interinstitutional Linkage Analysis: a Social Accounting Matrix Multiplier Approach for the Mexican Economy", Economic Systems Research, vol. 18, núm. 1.

Bracamonte, A., y R. Méndez (2004), “Aplicación de matrices de contabilidad social para el estudio de comunidades rurales. El caso de Trincheras y Arizpe, Sonora", Sociedades Rurales, vol. 5, núm. 8, UAm, México.

Cardenete, M., y F. Sancho (2003), "Evaluación de multiplicadores contables en el marco de una matriz de contabilidad social regional", Investigaciones regionales, núm. 2, Alcalá de Henares, Asociación Española de Ciencia Regional.

Chapa, J., et al. (2008), "Matriz de contabilidad social Nuevo León 2004", Desarrollo social en Nuevo León: Reflexiones en torno a la pobreza, desigualdad, migración, bienestar, consumo, y grupos vulnerables, Cuadernos del Consejo de Desarrollo Social 8, del Consejo de Desarrollo Social de Nuevo León.

Domínguez, M. (2008), "Efectos económicos de los programas sociales en el estado de Nuevo León. El caso del programa Oportunidades, Programa de Atención al Adulto Mayor y Programa de Apoyo para las Personas con Discapacidad", Cuadernos del Consejo de Desarrollo Social 9, del Consejo de Desarrollo social de Nuevo León.

Instituto Nacional de Estadística, Geografía e Informática (INEGI) (2006a) (citada el 8 de junio de 2006), http://cuentame.inegi.gob.mx/glosario/h.asp

_-(2006b), (citada el 22 de mayo de 2006), mapoteca digital del INEGI-2006, http:// cuentame.inegi.gob.mx

(2005), Anuario Estadístico del Estado de Coahuila, México. (2004), Encuesta Nacional de Ingreso y Gastos de los Hogares (ENIGH), México.

Pyatt, G., y J. Round (1979), "Social Accounting and Fixed Price Multipliers in a Social Accounting Matrix Framework", The Economic Journal, núm. 89.

— , y E. Thorbecke (1976), "Planning techniques for a Better Future”, Working Paper, ILO, Programa sobre Empleo Mundial.

Rasmussen, P. (1956), Studies in intersectoral relations, Einar Harcks Forlag \& NorthHolland Publishing Company, Copenhague y Amsterdam.

Secretaría de Agricultura, Ganadería, Desarrollo Rural, Pesca y Alimentación (2001), Análisis de la cadena de comercialización de la manzana en el municipio de Arteaga Coahuila.

Secretaría de Planeación y Desarrollo del Gobierno del Estado de Coahuila (2005), Perfil Municipal de Arteaga.

Stone, R. (1944), "National Income and Expenditure” (unknown binding).

Thorbecke, E. (1998), "Methods of Interregional and Regional Analysis", Working Paper, Ashgate Publishing Limited. 
Yúnez-Naude, A., y E. Taylor (1999), Manual para la elaboración de matrices de contabilidad social con base en encuestas socioeconómicas aplicadas a pequeñas poblaciones rurales. Centro de Estudios Económicos, El Colegio de México. (1999), "Impactos de los cambios económicos en el agro mexicano y en la migración: un análisis micro multisectorial”, documento de discusión, El Colegio de México.

(2001), "Las remesas y el desarrollo rural", Seminario Internacional sobre las Transferencias y Uso de las Remesas: Proyectos Productivos y de Ahorro, documento de discusión, El Colegio de México.

— y S. González (2008), "Efectos multiplicadores de las actividades productivas sobre el ingreso y pobreza rural en México", El Trimestre Económico, vol. Lxxv (2), núm. 298, FCE, México.

Villa, V. (2001), Análisis estructural de la economía del ejido El Manantial, municipio de Matamoros, Coahuila, tesis de maestría en economía con especialización en finanzas, Universidad Autónoma de Coahuila. 